\title{
BMJ Open How do Jordanian and Syrian youth living in Jordan envision their sexual and reproductive health needs? A concept mapping study protocol
}

\author{
Jewel Gausman, ${ }^{1}$ Areej Othman, ${ }^{2}$ Iqbal Lutfi Hamad, ${ }^{3}$ Maysoon Dabobe, ${ }^{3}$ \\ Insaf Daas, ${ }^{4}$ Ana Langer ${ }^{1}$
}

To cite: Gausman J, Othman A, Hamad IL, et al. How do Jordanian and Syrian youth living in Jordan envision their sexual and reproductive health needs? A concept mapping study protocol. BMJ Open 2019;9:e027266. doi:10.1136/ bmjopen-2018-027266

- Prepublication history for this paper is available online. To view these files, please visit the journal online (http://dx.doi. org/10.1136/bmjopen-2018027266).

Received 16 0ctober 2018 Revised 29 October 2018 Accepted 30 0ctober 2018

Check for updates

(c) Author(s) (or their employer(s)) 2019. Re-use permitted under CC BY-NC. No commercial re-use. See rights and permissions. Published by BMJ.

${ }^{1}$ Women and Health Initiative; Department of Global Health and Population, Harvard T.H. Chan School of Public Health, Boston, Massachusetts, USA

${ }^{2}$ School of Nursing, University of Jordan, Amman, Jordan

${ }^{3}$ Jordanian Hashemite

Foundation for Human

Development (JOHUD), Amman, Jordan

${ }^{4}$ Center for Women's Studies, University of Jordan, Amman, Jordan

Correspondence to

Dr Jewel Gausman;

jmg923@mail.harvard.edu

\section{ABSTRACT}

Introduction Youth in Jordan constitute $20.4 \%$ of the population, and many face considerable challenges in addressing their sexual and reproductive health (SRH) needs, such as those related to the prevention of unintended pregnancy and sexually transmitted infections, early marriage and sexual coercion and violence. The findings of previous studies indicate that Jordanian youth require reproductive health-related support, information and services; however, there remains very limited data as to how youth envision their SRH challenges and needs. This protocol outlines the design of a qualitative study using a participatory research methodology called concept mapping. This methodology enables participants to develop a conceptual framework for how they envision their sexual and reproductive health needs.

Methods and analysis This study will use concept mapping, which consists of a structured and iterative participatory research process that engages participants over three data collection sessions in order to generate the information needed to create a visual display of their ideas pertaining their SRH needs, issues and concerns, and how these ideas relate to each other. Each data collection session focuses on a different activity, including brainstorming, pile sorting and interpretation of the results. Data will be analysed using hierarchical cluster analysis and multidimensional scaling. Transcriptions of group discussions will be coded and analysed to add depth to the study results. Two hundred and eighty-eight males and females of Jordanian or Syrian descent living in Jordan will be recruited from four communities across Jordan.

Ethics and dissemination This study meets the requirements of the Declaration of Helsinki and has been approved by the Institutional Review Boards at the Harvard T.H. Chan School of Public Health and the University of Jordan. Study findings will be presented in peer-reviewed, international journals and made available to local programme managers, policy-makers and stakeholders through local dissemination efforts.

\section{INTRODUCTION}

The United Nations defines young people as those aged between 10 and 24 years; this includes adolescents (10-19 years) and youth
Strengths and limitations of this study

- The use of concept mapping, an iterative and interactive qualitative data collection and analysis technique, encourages participants to open up about sensitive topics-especially younger youth.

- This study includes young men; a population that is commonly overlooked in studies on sexual and reproductive health

- The sample size is robust $(n=288)$ for concept mapping in order to obtain a rich data set.

- While a strength of this study lies in the fact that sexual and reproductive health issues in this population are understudied, the cultural sensitivity of the topics discussed may influence the results in that participants may not be comfortable sharing their ideas.

- Because this study is qualitative in nature, results may not be generalisable outside the study population.

(15-24 years). ${ }^{1}$ During these years, individuals undergo fundamental physiological and psychosocial changes, often with limited reproductive health information. ${ }^{23}$ In less developed countries, the obstacles that stand in the way of a healthy transition to adulthood are the highest. ${ }^{4}$ The world's youth are at a higher risk of sexual and reproductive morbidity and mortality due to a variety of factors including early marriage, early pregnancy, unsafe abortion, sexually transmitted infections (STIs), harmful traditional practices and sexual coercion. ${ }^{5-7}$ Conservative sociocultural norms, such as those found in many areas throughout the Middle East, create barriers to youth obtaining specific, timely and high-quality sexual and reproductive health (SRH) information and services. ${ }^{89}$

Youth in Jordan constitute $20.4 \%$ of the population, ${ }^{10}$ many of whom face considerable challenges in addressing their SRH needs, ${ }^{2}{ }^{111}$ including those related to the 
prevention of unintended pregnancy and STIs, ${ }^{11-15}$ early marriage,${ }^{1617}$ and sexual coercion and violence. ${ }^{18} 19$ The findings of previous studies indicate that Jordanian youth need reproductive health-related support, information and services. The National Youth Survey conducted in 2001 included 2142 Jordanians aged $15-24 .{ }^{11}$ The survey found that $29 \%$ of women and $44 \%$ of men aged 15-24 did not know the meaning of the term reproductive health, and healthcare providers and media were the preferred sources of family planning (FP) and SRH information, compared with peers or parents. A recent qualitative study of 60 Jordanian adolescent men and women between the ages of 12 and 18 indicated that there is significant demand for reproductive health information, counselling and accessible, youth-friendly services near where they live or study. ${ }^{3}$

A few qualitative studies conducted among Jordanians and Syrians have documented that youth perceive available FP services to be unpleasant, unprofessional and of poor quality. ${ }^{30}$ Additionally, youth cite concerns that providers do not take them seriously, treat them like children, do not know what information youth need and view youth's questions as inappropriate. ${ }^{21}$ An evaluation of services offered at selected public and private clinics in Jordan noted a lack of private rooms at Ministry of Health $(\mathrm{MOH})$ clinics, ${ }^{22}$ which is a concern that has been echoed by youth. ${ }^{3}$ Another study found that providers often give incorrect information to youth regarding reproductive health, such as instructing them not to use contraception because of fears over the relationship between FP use and infertility. ${ }^{23}$ Because of these and other challenges, utilisation rates among adolescents of public sector health services are extremely low. The Jordanian $\mathrm{MOH}$ estimates a primary healthcare utilisation rate among adolescents of $1 \%$ or less. $^{24}$

Jordanian society is fairly conservative with regard to norms relating to sexuality, and the discussion of sex-related issues openly or within families is often unacceptable and taboo. Sexual relationships are often prohibited outside of marriage ${ }^{23}$ As a result of the culture of silence surrounding sexuality, youth in Jordan exhibit limited knowledge on SRH issues. Additionally, the cultural context around sexual and reproductive health and rights (SRHR) in Jordan renders the study of SRHR issues challenging, thus limiting the availability of data and its reliability. ${ }^{1825}$

In assessing the SRHR needs of youth in Jordan, it is important to note that approximately $30 \%$ of Jordan's population is non-Jordanian, with almost one half of them being Syrian refugees. Of the estimated 1.2 million Syrian refugees currently living in Jordan, ${ }^{10}$ only $16 \%$ reside in the five official camps. ${ }^{26} 27$ More than one-third live in the capital, one-third in Irbid, $16 \%$ in Mafraq and 14\% in Zarqa. ${ }^{28}$ The large population growth in many of these areas has significantly increased demand on the limited health resources available, while deepening existing socioeconomic deprivation, such as poverty, geographical isolation, gender-based discrimination and inadequate governance that ultimately manifests in further limiting access to SRH services. ${ }^{29}$

Syrian men and women living in Jordan face unprecedented challenges with regard to their SRH. The humanitarian crisis has caused young girls to become increasingly vulnerable to poor SRH outcomes, such as early pregnancy, child marriage, gender-based violence and sexual assault as a result of changing economic pressures, community dynamics and social norms. ${ }^{29-32}$ Previous qualitative research has highlighted that many young women fear negative repercussions, such as stigmatisation and discrimination from service providers and their communities, should they seek SRH services, while others report poor treatment by healthcare workers. ${ }^{33}$ Additionally, many Syrian refugee youth do not have basic knowledge related to reproductive health or know where to find a provider. ${ }^{34-37}$ A study conducted in 2013 among Syrian refugees in camps found evidence of both severe supply and demand side barriers, such as provider bias in offering women contraception and limited knowledge among women of where to obtain SRH services, resulting in reports of provider refusal to provide emergency contraception postrape and women reporting self-induced abortion because of the lack of contraceptive availability. ${ }^{38}$ Women living outside of camps often faced increased challenges with regard to SRH than those in camps, given that they tend to be younger, poorer and less educated. ${ }^{34-37} 39$

As Jordan's health system tries to respond to the changing needs in these communities, more research is needed that examines how youth envision their SRH concerns, as well as the challenges that they encounter related to meeting their reproductive health needs. In Jordan, the SRH health needs of youth are largely understudied due to concerns over the sensitive nature of discussing such topics with youth, and assumptions that conflate the silence on SRH issues with a lack of need. ${ }^{40}$ Many existing studies have limited sample sizes and other methodological limitations. ${ }^{231123}$ Furthermore, a majority of research on SRH among youth was conducted before the Syrian civil war. Our study will, therefore, make an important contribution to understanding SRH from the perspective of youth in Jordan, while also offering a comparative perspective between Syrians and Jordanians and by participant sex. Hence, with this study, we aim to address the following research questions:

1. How do youth define and prioritise their SRH needs in Jordan?

2. What are the differences in the SRH needs between Jordanians and Syrians, as well as between young men and women?

\section{METHODS AND ANALYSIS}

\section{Methodological overview}

This study uses an iterative and participatory qualitative research methodology called concept mapping. Concept mapping is a structured qualitative data collection and analytical process that ultimately results in the development 
of a visual conceptual framework that depicts how a group views key topics within a given conceptual domain and how the relationships between those topics are structured. ${ }^{41} 42$ The process of concept mapping typically occurs over a sequential series of group sessions that build on one another, which include interactive activities, such as brainstorming, pile sorting and group discussion. The final result is a visual map that depicts the conceptual framework developed by the group. Data collection activities are interspersed with an analytical process.

Previous studies have used concept mapping to engage youth in sensitive and abstract concepts, including those related to SRH. ${ }^{43}{ }^{44}$ One benefit of this approach, especially in its use with sensitive topics, is that its longitudinal nature enables youth to become more comfortable with the topic and the other participants over time. By applying this underused approach, this study will add to the literature on this methodology's use and relevance among adolescents.

\section{Participant selection and recruitment}

The current study will include Jordanian and Syrian adolescents (aged 15-19 years) recruited from the Jordanian cities of Amman, Irbid, Mafraq and Zarqa. In total, 288 participants will be enrolled in the study, assuming an attrition rate of $10 \%$. Recruitment of participants will be divided equally between study sites and stratified by gender and national origin. In each community, 18 Jordanian males, 18 Jordanian females, 18 Syrian males and 18 Syrian females will be recruited. Participants will be recruited by convenience from a local community development centre that provides health and livelihood programmes to underserved youth. Community meetings will be held to discuss the study in order to ensure support from the local community leaders, and participants will be invited to enrol in the study following those meetings.

The required number of participants for concept mapping varies according to the type of research ${ }^{45}$; however, the proposed number of study participants (288 participants) is robust compared with other studies using concept mapping. ${ }^{446}{ }^{47}$ We decided on a large number of participants because of the sensitive nature of the topic, the desire to compare data by sex and national origin, and to ensure that we reach saturation and generate a wide range of ideas for discussion.

\section{Data collection procedures and iterative analytical process}

Data collection will take place from September to November 2018. Youth will be asked to participate in three separate group sessions, each lasting 1.5 hours. The first group session will be scheduled at the time of recruitment. All three sessions will take place within a 3-week period. Each group session will include up to 18 individuals of the same sex and national origin. Group facilitators will be of the same sex as participants and will be from the same communities to ensure the participants feel comfortable sharing information.

Different activities will occur in each of the three data collection sessions. The importance of confidentiality and respect will be emphasised at the beginning of each session. At the beginning of the first session, the facilitator will brainstorm ground rules with the participants. Ground rules will be written on a large flip chart at the front of the room, and it will be reviewed at the start of all subsequent data collection sessions. Then, participants will be asked to complete a short demographic survey before starting activities. The demographic survey will be multiple choice and collect information on age, city of residence, nationality, educational status of the participant and his/her parents, marital status, age of marriage, perceived wealth, where the individual receives reproductive health information, and their previous experience with reproductive health service provision.

Participants will then be guided through a brainstorming session, in which participants' first work in pairs before rejoining the large group discussion. As a warm-up activity to initiate conversation, the facilitator will ask the group what is meant by the phrase SRH. After individuals have volunteered some ideas, the facilitator will provide the following definition: 'Good sexual and reproductive health is a state of complete physical, mental and social well-being in all matters relating to the reproductive system. It implies that people are able to have a satisfying and safe sex life, the capability to reproduce, and the freedom to decide if, when, and how often to do so. ${ }^{48}$ The facilitator will write this definition on a flip chart so that participants can refer to it during the discussion, as needed.

Next, the facilitator will ask participants to work in pairs to discuss anything that comes to mind related to the 'different SRH concerns, challenges, needs or fears, that (the participants) or other people in (their) community (their) age may have, 'with a goal of developing a list of all of the concerns related to SRH that affect youth in the study communities. Participants will be asked to write everything discussed on a sheet of paper. After $10 \mathrm{~min}$, the facilitator will open a large group discussion. Participants will be given the opportunity to share the factors they brainstormed, and encouraged to add more factors as the group conversation continues. Facilitators will take notes on a large flip chart at the front of the room. Before the close of the session, facilitators will pass out paper and ask participants to privately write down any other issues that they would like to contribute but were too embarrassed or nervous to share with the group to be placed in a box at the front of the room. After the end of the first session, the focus group facilitators will collect the notecards, paper and the flip charts, and return them to the study team's principal researchers.

The first analytical step in the process occurs between the first and second sessions. After the first session, study staff will consolidate the brainstormed factors from all groups, keeping the factors separated by sex. Study staff will remove duplicates and consolidate brainstormed items into a master list of all the ideas generated in all groups.

During the second group session, participants will be divided into pairs and given a stack of notecards. On each notecard, an item from a consolidated list of topics will be printed. Each pair will be given a complete set of cards 
including all items from the master list. Participants will be instructed to organise the items into piles that are meaningful to them. There are no rules for how to sort the items except (1) no item can be placed in a pile by itself and (2) all of the items cannot be placed into the same pile. Once the piles are created, participants will be asked to generate a name for each pile that represents the items that it contains. After this task is complete, participants will be asked to fill out a paper survey that contains a list of each of the items that they used in the sorting activity. Participants will be asked to rate each item on a scale of $1-5$, both in terms of importance to their SRH and how sensitive the item is in their communities.

The data obtained from the pile sorting exercise will be analysed using hierarchical cluster analysis. ${ }^{42}{ }^{45}$ First, participant responses will be encoded into binary similarity matrices which will be added together to create a combined matrix of all participants' data. Second, a Euclidean distance matrix will be calculated from the combined matrix. Multidimensional scaling will be used to determine the degree of similarity between items and to organise them into a spatial configuration. ${ }^{49}$ Scree plots and Shepard plots will determine the number of spatial dimensions to best represent the data. ${ }^{50}$ Based on this analysis, individual items will be grouped into clusters that represent high-level concepts on a visual map. The distance between any two points or clusters will represent their degree of similarity or difference, indicated by their closeness (or distance from) each other. The preliminary names given to cluster will be initially selected based on participant data. Separate maps will be generated for boys and girls. Additionally, basic descriptive statistics will be calculated using the ranking data, and disaggregated by sex and nationality.

In the third and final session, participants will be asked to interpret the results and discuss the differences between key demographic groups (males/females and Syrians/ Jordanians). The results from the previous analytical step are presented on a visual map that shows aggregated relationships between ideas in which the list of concepts is organised into related higher level clusters. Participants will be asked to discuss their reactions to the map, whether they agree with the cluster groupings and preliminary names, and their insights into the spatial organisation of the map. Additionally, the facilitator will present the ranking data in terms of sensitivity and importance. Participants will be asked to identify and discuss the differences between groups and why those differences exist.

After the last session, the visual maps will be finalised based on confirmation during the group discussion. For example, participants may recommend changes to the names of clusters based on the content of items they contain. The transcripts from the final group discussion will be thematically coded and used to add depth to the interpretation of the cluster maps. Participant quotations will be used to support the results of the cluster maps.

Only the first and third group sessions will be audio recorded. Only the third session will be transcribed. Notes will be taken by the interviewers during the discussions in order to augment the digitally recorded data.

Group facilitators will undergo extensive training in order to become comfortable with the research approach and to ensure adherence to study procedures. An initial 2-day training will be provided to group facilitators that includes both didactic and participatory elements. Throughout the training, group facilitators will participate in the concept mapping activities as though they are participants so that they may better internalise study activities and observe the facilitator's role. At the end of the second day of the initial training, facilitators will pretest the study materials with one group each of males and females in the target age range from one of the study communities prior to initiating data collection activities. This will provide an opportunity for the facilitators to practise study procedures. Additionally, it will provide an opportunity to revise any instruments, if needed. Finally, before the last data collection session, facilitators will again undergo an additional 1-day training that will help familiarise themselves with the study results, so that they may be better equipped to support the interpretation of the data.

\section{Patient and public involvement}

Prior to developing the research questions and study approach, we first engaged in a participatory process that included consultations with local policy-makers (including locally elected government officials and representatives from relevant government ministries), stakeholders (including representatives from donor organisations, non-governmental organisations and health service-delivery organisations active in Jordan), and members of the study population (Jordanian and Syrian youth aged 15-19 years). In addition to individual meetings, we held a consultation workshop in which participants from the groups identified above discussed our research objectives, questions and our proposed methodological approach. This consultation provided important insight as to how to guide our research so as to be most relevant to the local context and needs, and how to most effectively approach communities to ensure local buy-in given the sensitive nature of the study topic. Additionally, participants identified potential ethical concerns with participants and how to mitigate those issues. As part of this process, we also discussed preferences related to research utilisation and dissemination of our findings to ensure that our findings are presented to community stakeholders and participants in ways that would facilitate the integration of our results into policy and programmes.

Following the recommendations from the consultative process, we established a steering committee that consists of opinion leaders, academics and policy-makers in the fields of reproductive health, education and youth in Jordan. Additionally, we established a second steering committee that includes Jordanian and Syrian youth within the study's target age range (aged 15-19 years). The role of the steering committee is to provide ongoing review of study objectives, procedures and methodologies in order to ensure the research is contextually relevant and to provide 
guidance on any ethical concerns related to the research. A secondary objective of this steering committee is to cultivate ownership of the study procedures of results, and to develop strategies for effective dissemination of research results.

One of our priorities is to ensure that the results of our study are disseminated to study participants and their communities. One of the ways in which we will ensure that participants interact with the study's results is inherent to the research methodology itself. The iterative, participatory process that is central to concept mapping as a methodology enables study participants to actively engage with and react to their own data throughout the data collection process while also supporting efforts in data analysis. We also plan to also hold targeted events in order to ensure that the research results are disseminated more broadly within the study communities. We plan to host round table discussions that include members of the four study communities, and we will also engage members of a popular, local youth-led radio station to develop create ways to disseminate key messages from our study to a broad audience.

\section{RESEARCH ETHICS}

As the study involves both minors (under the age of 18) and those above the age of majority in Jordan (18 years and older), separate consent processes will occur for each age group. At the time of recruitment, minors will be given a parental permission sheet. Parents will be required to return to the sheet to study staff at the local community development centre in person, otherwise, their child will not be allowed to participate. Minors will undergo an informed assent process using simplified language on the day of the first data collection session, prior to its start. Participants 18 years of age and above will undergo informed consent at the time of recruitment.

To protect the confidentiality of the participants in the study, we will not collect direct, personal identifiers at any point in the study process. On enrolment, individuals will select an anonymous identifier by which to refer to themselves, and will refer to that codename in each of the focus group discussions so that we can track enrolment. There will be no documents that link the codename and any personally identifiable information. Facilitators will be instructed not to collect any personally identifiable information in any of their data collection materials. Audio recordings and other study materials will be destroyed once the data collection activities are complete.

Refugees included in this study will be recruited from those living outside of refugee camps. Some of the refugees recruited may be officially registered as such, and some may not. Because a refugee's registration status is somewhat sensitive in that it relates to access to government benefits and services, we will not ask about whether or not the refugee is registered as part of the recruitment process; however, we will provide information to refugees about the registration process should they be interested in learning more about registration. Refugees constitute a large percentage of the population in all the study communities. Given their large number, as well as the fact that many governmental and non-governmental organisations in the study communities work with refugees, their participation in this study is not expected to cause any additional risk. Furthermore, having an equal number of Jordanian nationals and refugees included across all phases of data collection ensures no discriminant conduct.

Data collection activities will occur in a community development centre that is run by a local non-governmental organisation active in community development. Each community development centre has private space so that confidentiality can be maintained. Considering it is common for youth and parents to regularly participate in events at these community development centres, anonymity will be maintained among study participants, as their presence at the sites will not raise any undue attention within their communities. All participants will be given information regarding where they can obtain health or other social support services, and facilitators will be available after each session so that participants can speak with them privately about any concerns.

\section{DISCUSSION AND DISSEMINATION}

This study will provide important insight for future research, policy and programmes intending to better serve youth in Jordan and across the Middle East. There is very limited data on the concerns that the youth in this region have in relation to their SRH. As this research methodology will provide a conceptual framework for how youth themselves envision and prioritise their concerns, the results will provide important information so that programmes may better meet the constellation of their needs. As many of the SRH issues in this domain are inter-related and interact with each other ${ }^{51}$ concept mapping is an ideal methodology to engage youth on this topic.

In order to ensure that study results are made available for integration into policy and programmes, we will disseminate the results of this study in several ways. Aggregate results and final publications will be disseminated to the community and local institutions where the research is conducted, as previously discussed. Any publications/ reports that are prepared using this data will also be shared with local government bodies and other stakeholders in Jordan. Finally, it is anticipated that these data will be used to develop peer-reviewed manuscripts to publish in academic journals.

Contributors All authors were involved in conceptualisation of the study design and development of study methods, instruments and protocol. JG wrote the first draft. A0, ID, ILH, MD and AL provided critical input and revisions. All authors approved of the final draft.

Funding This work is part of the Sexual and Reproductive Health Research Programme with project number W 08.560 .012 , which is financed by the WOTRO Science for Global Development of the Netherlands Organisation for Scientific Research (NWO).

Competing interests None declared.

Patient consent Not required. 
Ethics approval This study has been approved by the Institutional Review Boards at the Harvard T.H. Chan School of Public Health and the University of Jordan.

Provenance and peer review Not commissioned; peer reviewed for ethical and funding approval prior to submission.

Open access This is an open access article distributed in accordance with the Creative Commons Attribution Non Commercial (CC BY-NC 4.0) license, which permits others to distribute, remix, adapt, build upon this work non-commercially, and license their derivative works on different terms, provided the original work is properly cited, appropriate credit is given, any changes made indicated, and the use is non-commercial. See: http://creativecommons.org/licenses/by-nc/4.0/.

\section{REFERENCES}

1. UNICEF. Adolescence: A Time that Matters: UNICEF, 2002.

2. Almasarweh IS. Adolescent and youth reproductive health in Jordan: status issues policies and programs, 2003.

3. Khalaf I, Moghli FA, Froelicher ES. Youth-friendly reproductive health services in Jordan from the perspective of the youth: a descriptive qualitative study. Scand J Caring Sci 2010;24:321-31.

4. UNFPA and WHO for the Adolescent Working Group under EWEC. Technical guidance for prioritizing adolescent health, 2017.

5. Blum RW, Bastos FI, Kabiru CW, et al. Adolescent health in the 21st century. Lancet 2012;379:1567-8.

6. Glasier A, Gülmezoglu AM, Schmid GP, et al. Sexual and reproductive health: a matter of life and death. Lancet 2006;368:1595-607.

7. Bearinger LH, Sieving RE, Ferguson J, et al. Global perspectives on the sexual and reproductive health of adolescents: patterns, prevention, and potential. Lancet 2007;369:1220-31.

8. Langer A, Meleis A, Knaul FM, et al. Women and Health: the key for sustainable development. Lancet 2015;386:1165-210.

9. DeJong J, El-Khoury G. Reproductive health of Arab young people. BMJ 2006;333:849-51.

10. Department of Statistics [Jordan]. 2015 National census report: Department of Statistics [Jordan], 2016.

11. Johns Hopkins University Center for Communication Programs Jordanian youth survey: knowledge, attitudes and practices on reproductive health and life planning. 2001.

12. Mahafzah AM, Al-Ramahi MQ, Asa'd AM, et al. Prevalence of sexually transmitted infections among sexually active Jordanian females. Sex Transm Dis 2008;35:607-10.

13. Khoury S, Bata M, Issa A, et al. Contraception acceptability among a group of newly delivered mothers in Jordan. Jordan Medical Journal 1986;21:229-47.

14. Hijazi $\mathrm{HH}$. Factors affecting contraceptive use among women of reproductive age in northern Jordan: a framework for health policy action, 2012

15. Higher Population Council and Health Policy Project. Jordan National Reproductive Health/Family Planning Strategy: At a Glance, 2013.

16. Kradsheh M. Female early marriage: its determinants and effects on demographic behavior in Jordan. Jordan Journal of Social Sciences 2012;5.

17. UNICEF. A study on early marriage in Jordan. Amman, Jordan: UNICEF, 2014.

18. Okour AM, Badarneh R. Spousal violence against pregnant women from a Bedouin community in Jordan. J Womens Health 2011;20:1853-9.

19. QED Group. USAID/Jordan: Gender Analysis and Assessment, 2012.

20. Shakhatreh FMN. Family planning in women of childbearing age in disadvantaged south Jordan. European Journal of Contraception and Reproductive Health Care 2012;17:S72.

21. Khalaf I, Abu Moghli F, Froelicher ES. Youth-friendly reproductive health services in Jordan from the perspective of the youth: a descriptive qualitative study. Scand J Caring Sci 2010;24:321-31.

22. Okour AM, Saadeh RA, Zaqoul M. Evaluation of family planning counselling in North Jordan. Sultan Qaboos Univ Med J 2017;17:436.

23. Al-Shdayfat NM, Green G. Reflections on sex research among young Bedouin in Jordan: risks and limitations. Cult Health Sex 2012;14:101-11.
24. Ministry of Health of Jordan; UNFPA; and The National Women's Health Care Centre. An evaluation of comprehensive maternal, child and reproductive health services. 2015.

25. Damra JK, Abujilban SK, Rock MP, et al. Pregnant women's experiences of intimate partner violence and seeking help from health care professionals: a jordanian qualitative study. J Fam Violence 2015;30:807-16.

26. Doocy S, Lyles E, Roberton T, et al. Prevalence and care-seeking for chronic diseases among Syrian refugees in Jordan. BMC Public Health 2015;15:1097.

27. Achilli L. Syrian refugees in Jordan: a reality check, 2015.

28. UNHCR. Syrian Refugees Living Outside of Camps in Jordan: Home Visit Data Findings, 2013.

29. UNICEF. Equity for Children \& Adolescents in Jordan: A Country Profile, 2015.

30. Jordan Higher Population Council. The third national report on success in excuting 2013 cairo action plan for population and development planning for development beyond 2015. 2015.

31. Schmidt R. Meeting their needs: results from a Knowledge, Attitudes and Practices (KAP) survey on gender-based violence and reproductive health in the Mafraq and Irbid Governorates, Jordan. 2015.

32. Women UN. Gender-Based Violence and Child Protection Among Syrian Refugees in Jordan, with a focus on child marriage. 2013.

33. Higher Population Council. Reproductive health services provided to Syrians living outside camps in Jordan, 2016.

34. Jordan Communication Advocacy and Policy Activity. Family Planning among Syrian Refugees in Jordan, 2016.

35. VAF Assessment Team. Vulnerability Assessment Framework Baseline Survey. Amman, Jordan: UNHCR, 2015.

36. UNHCR. Health Access and Utilization Survey Among Non-Camp Syrian Refugees: UNHCR, 2014

37. UNHCR. Health Access and Utilization Survey Among Non-Camp Refugees in Jordan Final Report, 2015.

38. Krause S, Williams H, Onyango MA, et al. Reproductive health services for Syrian refugees in Zaatri Camp and Irbid City, Hashemite Kingdom of Jordan: an evaluation of the Minimum Initial Services Package. Confl Health 2015;9:S4.

39. Ravishankar; N, Gausman J. Analysing equity in health utilization and expenditure in Jordan with focus on maternal and child health services. 2016.

40. Ercevik Amado L. Sexuality and Sexual Rights in Muslim Societies. Development 2009;52:59-63.

41. Trochim WMK. An introduction to concept mapping for planning and evaluation. Eval Program Plann 1989;12:1-16.

42. Burke JG, O'Campo P, Peak GL, et al. An introduction to concept mapping as a participatory public health research method. Qual Health Res 2005;15:1392-410.

43. Davis TS, Saltzburg S, Locke CR. Assessing community needs of sexual minority youths: modeling concept mapping for service planning. J Gay Lesbian Soc Serv 2010;22:226-49.

44. Bayer AM, Cabrera LZ, Gilman RH, et al. Adolescents can know best: using concept mapping to identify factors and pathways driving adolescent sexuality in Lima, Peru. Soc Sci Med 2010;70:2085-95.

45. Kane M, Trochim WM. Concept mapping for planning and evaluation. Sage 2007.

46. Visek AJ, Achrati SM, Mannix H, et al. The fun integration theory: toward sustaining children and adolescents sport participation. $J$ Phys Act Health 2015;12:424-33.

47. Baskin ML, Dulin-Keita A, Thind $\mathrm{H}$, et al. Social and cultural environment factors influencing physical activity among africanamerican adolescents. J Adolesc Health 2015;56:536-42.

48. United Nations Population Fund. Sexual and reproductive health, 2018

49. Anderberg MR. Cluster analysis for applications: probability and mathematical statistics: a series of monographs and textbooks: Academic press, 2014

50. Steyvers M. Multidimensional scaling. Encyclopedia of Cognitive Science 2002.

51. Clark CJ, Spencer RA, Khalaf IA, et al. The influence of family violence and child marriage on unmet need for family planning in Jordan. J Fam Plann Reprod Health Care 2017;43:105-12. 